\title{
Automédication : l'influence du contexte local dans les perceptions et pratiques des médecins généralistes
}

Stéphanie Larramendy et Sébastien Fleuret

\section{(2) OpenEdition}

\section{Journals}

Édition électronique

URL : https://journals.openedition.org/rfst/472

DOI : $10.4000 /$ rfst.472

ISSN : 2492-3672

Éditeur

Espaces et SOciétés (UMR 6590)

Référence électronique

Stéphanie Larramendy et Sébastien Fleuret, « Automédication : I'influence du contexte local dans les perceptions et pratiques des médecins généralistes », Revue francophone sur la santé et les territoires [En ligne], Miscellanées, mis en ligne le 13 mai 2015, consulté le 21 septembre 2021. URL : http:// journals.openedition.org/rfst/472 ; DOI : https://doi.org/10.4000/rfst.472

Ce document a été généré automatiquement le 21 septembre 2021.

La Revue francophone sur la santé et les territoires est mise à disposition selon les termes de la Licence Creative Commons Attribution - Pas d'Utilisation Commerciale - Partage dans les Mêmes Conditions 4.0 International. 


\title{
Automédication : l'influence du contexte local dans les perceptions et pratiques des médecins généralistes
}

\author{
Stéphanie Larramendy et Sébastien Fleuret
}

1 Le médicament est un sujet relativement peu étudié sous l'angle géographique. Néanmoins il existe des études sur les variations dans l'usage et la consommation de médicaments (Bussi \& Dab, 1996; Nordmann, 2012), la répartition des officines et la distribution des médicaments sur le territoire (Devictor \& Sambuc, 2002), les variations géographiques dans l'accès aux médicaments (Gateaux \& Heitz, 2008), ou encore la place du médicament dans la circulation des pratiques de soins et des patients (Bochaton, 2014). L'automédication en revanche, n'a pas encore fait l'objet d'une étude qui adopte une lecture territorialisée. C'est ce que propose cet article.

2 Une étude réalisée par l'IPSOS en 2005 pour la Caisse Nationale d'Assurance Maladie faisait état de la surconsommation de médicaments des Français par rapport à leurs voisins européens, principalement en lien avec le nombre de médicaments prescrits à chaque consultation médicale (IPSOS 2015). Le marché de l'automédication en France se distingue quant à lui par sa faible importance, en valeur comme en volume (Coutinet et Abecassis 2007). Les pouvoirs publics, dans un souci de maîtrise des dépenses de santé, cherchent toutefois à encourager ces pratiques d'auto-soins. Le déremboursement de nombreux médicaments, depuis 2003, consécutif à leur classement en « service médical rendu insuffisant » est considéré comme une ouverture de ces médicaments au marché de l'automédication. Si $68 \%$ des Français se déclarent favorables à l'automédication (Deloitte / Harris Interactive 2013), cette pratique n'est pourtant pas sans poser question. Notamment celle de savoir si l'automédication représente un danger. Le déremboursement de certains médicaments fait que ces derniers sortent du champ de la prescription médicale pour entrer dans celui de l'automédication, ce qui induit pour le patient d'une part un surcoût qui peut engendrer des inégalités et d'autre part le 
besoin d'une éducation thérapeutique qu'il ne trouvera plus dans le conseil du médecin si ce médicament ne relève plus de la prescription (Reynaud, 2008).

3 Le «rapport Coulomb " commandité en 2007 par le Ministre de la Santé désigne le médecin généraliste et le pharmacien comme les garants du «bon usage » des médicaments d'automédication et de la " prévention de certains risques encourus par le patient» (Coulomb et Baumelou 2007). Le rapport précise le devoir du médecin de "vérifier, voire d'orienter l'automédication du patient » au cours des consultations, précisant toutefois qu'il ne pourrait "être tenu responsable du mésusage d'un patient ». L'automédication, que l'on définira comme l'acte de consommer de sa propre initiative, un médicament sans consulter un médecin pour le cas concerné (Fainzang 2012), place donc le médecin généraliste au cœur d'un antagonisme, la volonté d'autonomie des usagers confrontée à celle d'encadrement par les pouvoirs publics, et l'oblige à se positionner face à des pratiques dont, par essence, il est exclu par le patient.

4 Les praticiens apparaissent réticents, voire hostiles, à ces comportements qu'ils peuvent percevoir comme déviants ou dangereux (Pouillard 2001, Fainzang 2006). Mais les positions des généralistes face à l'automédication sont sujettes à des variations importantes, notamment spatiales. On peut, par exemple, observer des différences d'approche vis-à-vis de l'automédication entre les médecins généralistes exerçant en milieu rural et ceux qui exercent en milieu urbain (Ventelou et Rolland 2009, Thay 2013). Plus que tout autre spécialiste, les généralistes ont un exercice territorialisé (Schweyer, 2004). Ils pratiquent en un lieu qui ne leur a pas été imposé par les pouvoirs publics (liberté d'installation) et soignent les personnes dans leur environnement «familial, communautaire, culturel» (Europe 2002). La pluralité des contextes territoriaux nous fait donc envisager la pluralité des positionnements chez ces professionnels de santé.

5 L'étude des variations dans les perceptions et pratiques des généralistes vis-à-vis de l'automédication repose sur trois questionnements: Comment les médecins généralistes intègrent-ils l'autonomie de leur patient à leur pratique? Comment encouragent-ils ou freinent-ils l'automédication dans l'exercice de leur profession? Et plus généralement, comment se positionnent-ils dans cette problématique ? Cet article tente de répondre à ces questions en s'appuyant sur une méthode de contextualisation des discours qui vise à replacer les pratiques médicales dans leur territoire en tant qu'espace à la fois physique et socialisé, c'est à dire à l'instar de Turco (1985) repris par Di Meo, (1990) en recherchant une objectivation sociale de l'espace vécu par les individus . Cette approche comporte plusieurs prérequis.

- Le territoire est une formation socio-spatiale délimitée à la fois par des représentations individuelles et collectives partagées (Di Méo, op. cit.) et par un certain nombre d'instances géographiques, économiques et politiques (il est fait référence ici aux différents niveaux et découpages territoriaux administratifs).

- Les variations géographiques dans les taux et les formes de recours à l'automédication peuvent alternativement être révélatrices ou constitutives d'inégalités territoriales de santé. Or ces inégalités ont, jusqu'à présent été essentiellement étudiées à l'aune des données de mortalité, de morbidité, d'espérance de vie ou de recours aux soins médicaux que ce soit dans les atlas de santé (Salem, Rican, Jougla, 1999), les travaux des observatoires régionaux de santé (Trugeon et al. 2006) ou ceux de la Datar (Vigneron E., 2011). Les pratiques d'autosoins n'ont pas, à notre connaissance, fait l'objet d'une étude géographique et cet article ouvre donc un nouveau champ dans le prolongement de l'évolution de la géographie de la 
santé décrite par Kearns et Moon (2002) comme portant un intérêt accru pour des modèles sociaux plus larges de la santé et des soins de santé.

- Il est important « d'appréhender différents niveaux d'analyse simultanément afin de saisir les disparités spatiales de santé » (Picheral 2001), « la distance et la proximité » étant des notions relatives, et un individu ne pouvant «exploiter que les ressources dont il a connaissance dans son espace de vie » (Fleuret et Séchet 2006), notamment l'offre sanitaire et sociale. Il s'agit donc de choisir les lieux et échelles d'analyse en fonction d'une échelle conceptuelle» (Philibert, Breton, 2007). Ici l'échelle retenue est celle d'agrégats de communes appartenant à des bassins de vie aux pratiques quotidiennes similaires (même pôle urbain majeur de référence, mêmes formes de mobilités (domicile-travail, chalandise, loisirs) et aux profils socio-résidentiels caractéristiques (voir plus loin).

- Suivant l'exemple de travaux visant à l'étude des médecines alternatives et complémentaires dans une lecture géographique, il s'agit d'explorer le rôle de l'espace et des lieux dans le contenu et les pratiques de l'automédication et d'examiner également des espaces nonphysiques qui peuvent être présents dans l'automédication, espaces imaginés et visualisés dans les traitements (Andrews et al., 2011).

6 Le travail présenté dans cet article, et conduit de février à août 2014 dans le cadre du programme de recherche ANR AUTOMED (voir plus loin) sur les déterminants de l'automédication, repose sur une série d'entretiens menés auprès de médecins généralistes choisis après un travail préalable d'analyse spatiale afin de sélectionner un terrain d'étude pertinent pour l'analyse principale. Un terrain appelé « miroir » a été sélectionné selon le même procédé pour le contrôle des informations recueillies (permettant notamment de distinguer les effets de contextes des invariants géographiques). Ce travail analytique préalable à la réalisation des entretiens est présenté dans la première partie de l'article.

7 Cette recherche repose sur l'analyse des perceptions et pratiques de médecins généralistes $(\mathrm{N}=30)$ recueillis par des entretiens semi-directifs. Elle se concentre principalement sur les médecins généralistes, et s'enrichit de l'analyse de l'expérience et des perceptions des pharmaciens $(\mathrm{N}=19)$ implantés sur les mêmes territoires d'étude, les pharmaciens étant des acteurs incontournables de la distribution du médicament en France.

8 La deuxième partie de cet article qui expose les principaux résultats de l'étude de terrain démontre l'intérêt de l'étude des données socio-spatiales sur les territoires d'exercice des médecins pour mieux comprendre leurs postures et pratiques au regard de l'automédication. Par extension, ces résultats permettent de mieux comprendre les comportements individuels vis-à-vis de l'automédication car ces comportements sont largement influencés par la nature de leur relation au médecin traitant. Ces résultats sont mis en discussion dans une troisième partie conclusive.

\section{Méthodologie}

La méthodologie de travail adoptée se décline en plusieurs temps. Premièrement, le choix du terrain d'étude et de son terrain miroir repose sur des outils d'analyse spatiale à partir de données d'enquête et de statistiques publiques (INSEE). Deuxièmement, le travail de terrain est basé sur des entretiens semi-directifs conduits sur la base d'une grille d'entretien construite en interdisciplinarité par une équipe de médecins, 
sociologues et géographes. Ces deux étapes ainsi que les outils mis en œuvre sont décrits successivement ci-après.

\section{Le choix du terrain d'étude}

10 Ce travail s'inscrit dans le programme ANR AUTOMED, «Automédication choisie ou subie » 2013-2016, qui propose l'analyse des déterminants socio-économiques, sociaux, territoriaux, et pathologiques du recours à l'automédication, dans les départements de Loire Atlantique et de Vendée. Il mobilise conjointement des chercheurs et des médecins du Département de Médecine Générale de Nantes, des chercheurs sociologues du CENS (Nantes) et des géographes du laboratoire ESO d'Angers. Les méthodologies proposées y sont diverses, avec des études menées à partir de journaux de santé ou de questionnaires patients, ou bien encore à partir d'observations de consultations médicales. Notre étude participe à l'approche géographique de la problématique.

11 Il semblait pertinent de choisir des terrains d'étude sur lesquels des comportements différenciés en matière d'automédication apparaissaient. Nous avons défini dans un premier temps un territoire global d'investigation à partir de la géolocalisation des cabinets de médecine générale de l'étude quantitative sur les pratiques d'automédication du programme AUTOMED « Se Soigner Par Soi Même » (SSPSM), dans lesquels des questionnaires étaient auto-administrés par des patients. Ces questionnaires anonymes ont été distribués pendant 2 mois, dans des salles d'attente de cabinets par des internes en médecine de Nantes (54 internes) qui y effectuaient leur stage. Cette enquête qui interroge les pratiques d'automédication des répondants au cours des 6 mois précédents contient des informations utiles sur la situation socioéconomique et des données relatives aux espaces de vie des personnes enquêtées (lieux de résidence, d'achats, de loisirs, distance au cabinet, à la pharmacie). Cette enquête était destinée à se répéter 4 fois de 2012 à 2014 . Notre méthodologie s'appuie sur les données de la première vague réalisée de mai à juin 2013. Même si les vagues d'enquête successives n'étaient pas achevées, ce matériel constitue un excellent support pour l'analyse spatiale des comportements d'automédication.

12 Dans un second temps, nous avons réalisé un découpage du territoire défini en huit zones de plus petites tailles pour faire apparaitre des nuances spatiales dans les résultats des études quantitatives du programme AUTOMED. Pour effectuer le découpage, les communes ont été regroupées par bassins de vie, « plus petits territoires sur lequel les habitants ont accès aux équipements et services les plus courants " (INSEE 2010), eux-mêmes regroupés avec un souci de cohérence à partir des connaissances empiriques sur les habitudes de vie et les pratiques de mobilités régulières d'un bassin à un autre, et de l'obtention d'un effectif minimal de 50 questionnaires de la vague 1 de l'enquête SSPSM par zone (fig. 1, fig. 2). 
Figure 1 : Zonage à partir du territoire d'étude du programme AUTOMED (Arcgis)

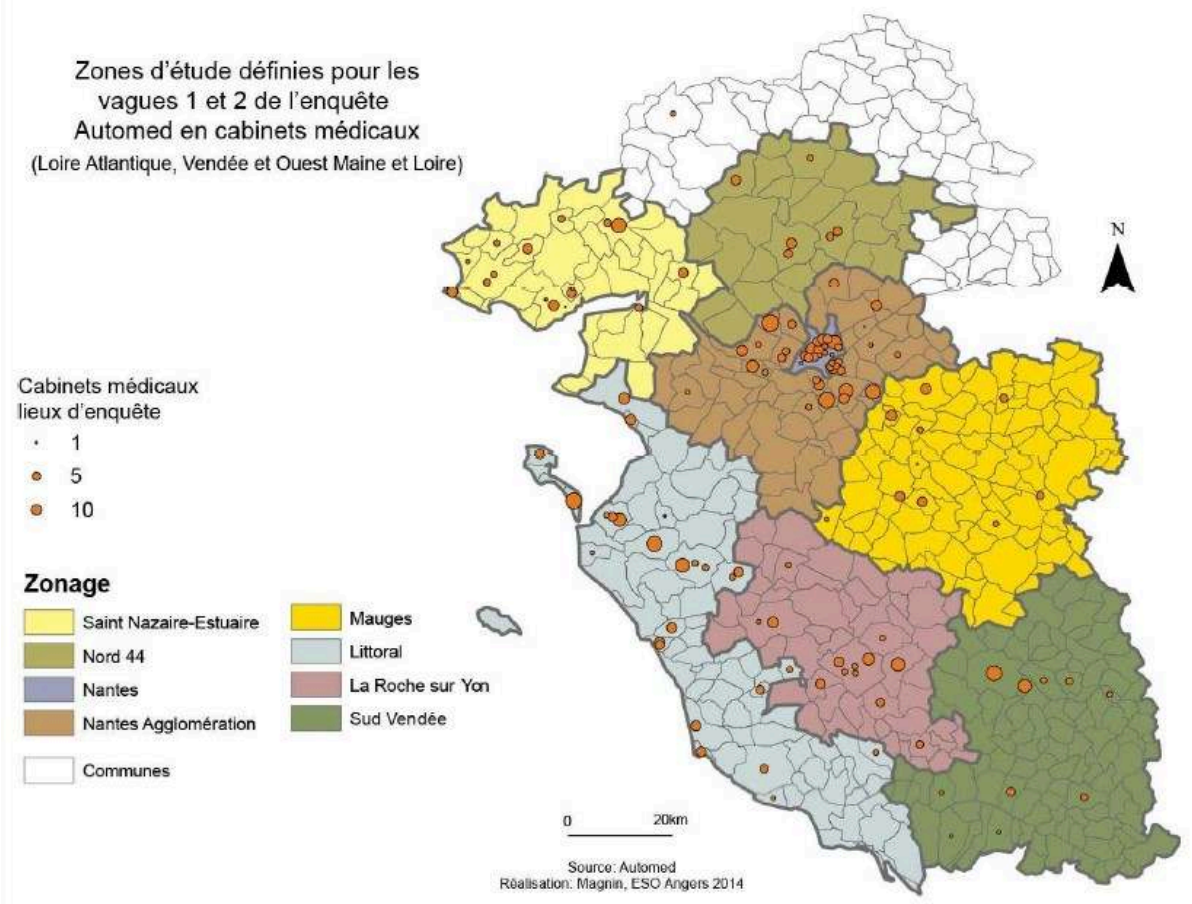

13 Ce découpage s'affranchit des limites des bassins de santé définis par le schéma régional d'organisation sanitaire. Ce choix est justifié par le fait que les cabinets médicaux participant à l'enquête ne couvrent pas l'ensemble du territoire. L'analyse géographique repose donc bien sur des périmètres définis à partir du matériel empirique et non à partir de limites administratives.

Figure 2 : Effectifs de questionnaires par zone

\begin{tabular}{|c|c|}
\hline Zones & Nombre de questiommaires par zone \\
\hline La Roche-sur-Yom & 56 \\
\hline Les Manes & 64 \\
\hline Lonoril & 190 \\
\hline Namtes & 102 \\
\hline Names agcloménation & 242 \\
\hline Nond 44 & 60 \\
\hline Suint Nazxire-Estuaire & 83 \\
\hline Sud Verulée & 92 \\
\hline
\end{tabular}

14 Le code attribué à chaque zone a été reporté dans la base de données des questionnaires de la vague 1 de l'étude quantitative SSPSM. Les taux d'automédication ont été calculés à partir de la question "Au cours des 6 derniers mois, avez-vous souffert d'au moins un problème de santé que vous avez soigné par vous-même, sans avoir besoin de consulter votre médecin?». 57,9\% des patients répondent oui. Conformément aux données de la littérature (Raynaud 2008, Laure 1998), les femmes plus que les hommes et la population d'âge actif plus que les retraités ont recours à l'automédication. De ce fait, les taux de recours à l'automédication sur chaque zone ont 
été standardisés par la méthode directe de standardisation sur l'âge et le genre pour éviter ce biais d'analyse. Un test statistique (Loi de Poisson) a été pratiqué pour établir les taux significativement différents de la moyenne. Les résultats sont présentés dans la figure 3.

Figure 3 : Taux standardisés sur l'âge et le genre d'automédication par zone

\begin{tabular}{|l|l|c|}
\hline \multicolumn{1}{|c|}{ Zone } & \multicolumn{1}{|c|}{$\begin{array}{c}\text { Taux } \\
\text { d'automédication } \\
\text { standardisé sur le } \\
\text { sexe et l'âge }\end{array}$} & p \\
\hline $\begin{array}{l}\text { Saint-Nazaire- } \\
\text { Estuaire }\end{array}$ & 42,8 & 0,08 \\
\hline Nord 44 & 59,2 & 0,07 \\
\hline Nantes & 70,3 & 0,06 \\
\hline Nantes \\
agglomération & 59,0 & 0,99 \\
\hline Les Mauges & 54,6 & 0,68 \\
\hline Littoral & 55,1 & 0,20 \\
\hline La Roche Sur Yon & 56,7 & 0,34 \\
\hline Sud Vendée & 51,3 & 0,40 \\
\hline
\end{tabular}

Trois zones se distinguent avec des taux de recours à l'automédication qui s'écartent de la moyenne en limite de signification statistique $(p<0,08)$, Saint-Nazaire-Estuaire avec les taux d'automédication les plus faibles du territoire, et Nantes et Nord 44 avec les taux de recours les plus importants. Nous pouvons ainsi définir deux territoires d'étude potentiels, Saint-Nazaire-Estuaire d'un côté et un territoire plus large allant de Nantes jusqu'aux communes du « Nord 44 » de l'autre.

16 La deuxième étude sur laquelle s'appuie le travail, a été réalisée à partir d'observations directes de consultations de médecins généralistes maîtres de stage universitaire, en Vendée et Loire Atlantique, du 14 mai au 15 juin 2012 (Thay 2013) par des internes de médecine générale qu'ils accueillaient dans le cadre du stage " praticien » de niveau 1 du Diplôme d'études spécialisées de médecine générale. Ce travail de recherche cherchait à déterminer au travers des discussions entre le médecin et son patient :

1. si la question de l'automédication était abordée lors de consultations pour des pathologies aiguës,

2. qui initiait alors la discussion et si cette discussion était prise en compte dans les prescriptions.

Les médecins, s'ils connaissaient la thématique générale, n'étaient pas informés de l'objectif principal de cette étude afin de ne pas modifier leurs comportements. 126 médecins généralistes libéraux de Loire Atlantique et de Vendée, dont 47 femmes et 
79 hommes, ont participé à l'étude. L'enquête a porté sur 1597 rencontres de soins. $68,2 \%$ ont fait l'objet d'une discussion autour de l'automédication.

Les données de la base des observations de consultations de médecine générale ont été interrogées par zone (codage sur le code INSEE de la commune des cabinets médicaux). Les taux d'évocation de l'automédication soit par le questionnement du médecin, soit spontanément par le patient, et de sa prise en compte dans la prescription ont été calculés pour chacune d'elle. Un test statistique (Loi de Poisson) a ensuite été appliqué pour déterminer les taux significativement différents de la moyenne ( $\mathrm{p}<0,005)$ (fig. 4).

Figure 4 : Prise en compte de l'automédication des patients par les médecins généralistes par zone

\begin{tabular}{|l|l|l|l|l|l|l|}
\hline \multicolumn{1}{|c|}{ Zone } & $\begin{array}{c}\text { Taux de } \\
\text { mention par } \\
\text { le patient }\end{array}$ & \multicolumn{1}{|c|}{$\mathbf{p}$} & $\begin{array}{c}\text { Taux } \\
\text { d'exploration } \\
\text { par le médecin }\end{array}$ & \multicolumn{1}{|c|}{$\mathbf{p}$} & $\begin{array}{c}\text { Taux de prise en } \\
\text { ompte dans la } \\
\text { prescription }\end{array}$ & \multicolumn{1}{|c|}{$\mathbf{p}$} \\
\hline $\begin{array}{l}\text { Saint-Nazaire- } \\
\text { Estuaire }\end{array}$ & 53,4 & 0 & 66,5 & $<0,001$ & 70,6 & $0,0,001$ \\
\hline Nantes & 47,1 & 0,2 & 54,8 & 0,22 & 60,0 & 0,29 \\
\hline Nord 44 & 43,8 & 0,3 & 44,6 & 0,19 & 47,2 & 0,46 \\
\hline $\begin{array}{l}\text { Nantes } \\
\text { Agglomération }\end{array}$ & 39,7 & 0,2 & 45,7 & 0,13 & 49,9 & 0,25 \\
\hline Les Mauges & 41,9 & 0,5 & 43,0 & 0,21 & 54,8 & 0,001 \\
\hline Littoral & 36,0 & 0,1 & 55,7 & 0,07 & 36,3 & 0,22 \\
\hline La Roche sur Yon & 42,5 & 0,4 & 39,7 & 0,03 & 46,0 & 0,26 \\
\hline Sud Vendée & 38,2 & 0,3 & 40,9 & 0,11 & 45,5 & \\
\hline
\end{tabular}

Source : Observation de consultations de médecine générale, Thay, 2013

Les pratiques sur la zone de Saint-Nazaire-Estuaire se singularisent par des taux d'évocation, d'exploration et de prise en compte de l'automédication tous significativement supérieurs à la moyenne du territoire.

Ce travail de zonage a permis de faire apparaître des disparités territoriales à la fois dans les pratiques des patients et dans les pratiques des médecins généralistes vis-à-vis de l'automédication en Loire Atlantique et en Vendée. Le territoire de Saint-NazaireEstuaire se singularise particulièrement à la fois par les faibles taux d'automédication obtenus et par la plus grande prise en compte de cette problématique durant les consultations de médecine générale. Ce territoire, qui se compose principalement de communes situées le long de l'embouchure de la Loire et sur la côte Atlantique, présente donc un intérêt particulier pour notre approche qualitative. Les communes concernées sont toutes classées en « urbain » ou " périurbain » selon le zonage en aires urbaines INSEE $2010^{1}$. Les données de la littérature (Thay, 2013 ; Ventelou et Rolland, 2009) vont dans le sens de comportements différents des médecins généralistes vis-àvis de l'automédication en fonction du milieu d'exercice, opposant contexte urbain et contexte rural. Le choix d'un "miroir» sur des communes appartenant aux mêmes classes que celles de la zone focus apparaissait donc nécessaire pour s'affranchir du biais d'interprétation lié à des contextes trop différents. Cette étude en «miroir » a été 
réalisée sur Nantes et Nantes Nord, zones se singularisant également dans les taux de recours à l'automédication des patients. Cette démarche devait permettre de mettre en avant ce qui relève des spécificités du territoire de l'aire d'emploi nazairienne, au regard de données qui pourraient être plus sociologiques ou liées au contexte urbain en général.

\section{Le choix des communes au sein des territoires sélectionnés}

Travailler à l'échelle de la commune permet de contextualiser les discours. Afin d'en sélectionner un nombre limité par zone, les caractéristiques socio-économiques des communes concernées ont été comparées sur la base d'une classification en classes ascendantes hiérarchiques. L'analyse par classification ascendante hiérarchique consiste à opérer une répartition en sous-contextes : les communes ont été regroupées par étapes, deux à deux, en fonction des similitudes qu'elles présentent, jusqu'à l'obtention d'un découpage entre groupes de communes caractérisés par leur homogénéité interne et par des différences de l'un à l'autre. Pour cette analyse, huit variables socio-économiques issues de données INSEE 2010 ont été prises en compte: les taux de chômage, les taux d'ouvriers et de cadres, les niveaux de diplôme obtenu (BEP et inférieur, bac $+3, \mathrm{Bac}+5$ et plus), les taux de 65 ans et plus, le revenu médian par habitant. Les contextes ainsi dégagés par la classification ascendante hiérarchique sont caractérisés par des indicateurs socio-économiques plus ou moins favorables (fig. 5).

Figure 5 : Classification en classes ascendantes hiérarchiques des communes de Loire Atlantique et de Vendée selon des données socio-économiques INSEE 2010.

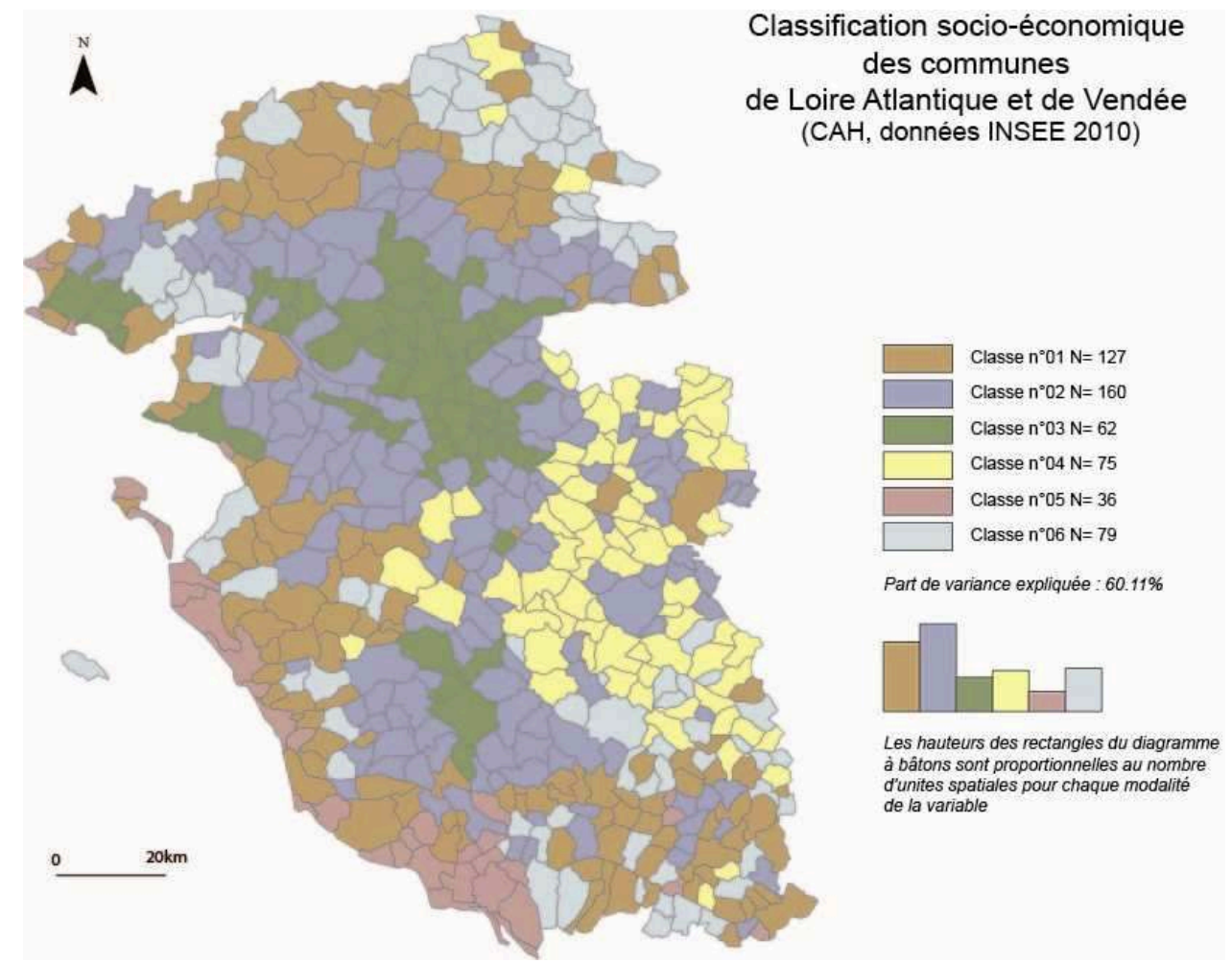

Cette classification en 6 types met à jour des différences socio-économiques dans les deux territoires d'étude qui seront à prendre en compte dans la lecture des résultats. Les communes en marron (classe 01) appartiennent à ce qui pourrait s'apparenter à 
une moyenne inférieure des communes du territoire sur les critères socio-économiques retenus. Les communes en vert (classe 03) présentent de forts taux de cadres, de diplômés des études supérieures, avec un revenu médian par habitant le plus élevé des deux départements. Les communes en bleu ciel (classe 06) sont caractérisées à la fois par une faible qualification de la population, un fort taux d'ouvriers, un taux de chômage supérieur à la moyenne et un revenu médian par habitant le plus faible du territoire. Celles en bleu foncé (classe 02) appartiennent à la moyenne "supérieure " des deux départements. Les communes en jaune (classe 04) et violet (classe 05) ne sont pas représentées dans nos terrains d'étude et correspondent respectivement à des communes avec de forts taux d'ouvriers et de faibles taux de chômage et des communes aux revenus médians par habitants élevés en lien avec une population de retraités socio-économiquement favorisés et de forts taux de chômage.

Les communes choisies comme terrain d'étude pour l'approche qualitative devaient comporter des cabinets ayant participé à la vague 1 de l'étude SSPSM et appartenir préférentiellement à des classes différentes (selon notre classification) afin de pouvoir mesurer le poids des données socio-économiques dans les discours. Dans la zone de Saint-Nazaire les communes de Guérande (classe 03), Saint-Nazaire, Paimboeuf (classe 01) et Trignac (classe 06) sont retenues et celles de Nantes, Saint Herblain, Sainte Luce, Héric (classe 03) et Nort sur Erdre (classe 02) dans la zone de Nantes.

L'analyse du contexte socio-économique, historique, sanitaire est ensuite approfondi sur ces communes pour pouvoir contacter les professionnels dans des quartiers de types différents (zone urbaine sensible, quartier pavillonnaire, ...) et mettre en perspective les discours avec le territoire d'exercice (cf. présentation du terrain d'étude). Ce travail s'appuie sur des données de la littérature, ainsi que sur des entretiens avec des acteurs locaux (Coordinatrice du Centre Communal d'Actions Sociales, travailleurs sociaux de l'APIE, médecins de la Permanence d'accès aux soins, du Conseil Général).

\section{Les entretiens semi directifs}

Les médecins généralistes ont été sollicités par téléphone pour réaliser des entretiens d'une vingtaine de minutes à leur cabinet. Les pharmacies enquêtées ont ensuite été sélectionnées en fonction de leur proximité aux cabinets médicaux retenus afin de pouvoir recouper les éléments contextuels. D'autres pharmacies ont été contactées sur le critère " pharmacie de passage ", c'est-à-dire dont la clientèle ne correspond pas à la population d'une zone territoriale déterminée (pharmacies de galerie marchande par exemple). Les entretiens avec les pharmaciens se sont déroulés à l'officine.

Des grilles d'entretien spécifiques à chaque type de professionnel ont été utilisées afin de contextualiser les propos (choix d'installation, données sociologiques, patientèle, ...) et d'identifier les perceptions et les pratiques de ces professionnels vis-à-vis de l'automédication (la définir, l'encourager ou la freiner, positionnement par rapport aux politiques publiques, réactions face à cette automédication, ...). Les pharmaciens ont également été interrogés sur leurs stratégies "marketing» vis-à-vis de l'achat de médicaments hors prescription. La quasi totalité des entretiens a été réalisée par la même enquêtrice pour ne pas introduire de biais dans le questionnement des différents professionnels. Les entretiens, enregistrés après accord, ont ensuite été intégralement retranscrits pour permettre une analyse fine des discours grâce au logiciel Nvivo. Les 
différents thèmes définis dans les grilles d'entretien et repris au cours des entretiens ont été encodés puis analysés pour leur contenu, sans comptage de la récurrence des mots.

27 En tout, 30 médecins généralistes et 19 pharmaciens ont été enquêtés au cours d'entretiens allant de 20 minutes à 1 heure. Les taux de refus ont été équivalents entre les deux zones étudiées. Sur la zone de Saint-Nazaire-Estuaire, 16 médecins généralistes ont accepté de participer, dont 11 femmes $(68,75 \%)$ et 5 hommes $(31,25 \%)^{2}$, répartis sur les communes de Guérande (2), Saint-Nazaire (8), Trignac (5, soit l'ensemble des médecins de la commune) et Paimboeuf (1). 13 sont en cabinet de groupe. La moyenne d'âge était de 50,6 ans [33;63]. 11 pharmaciens ont été enquêtés, dont 2 à Guérande, 6 à Saint-Nazaire, 2 à Trignac et 1 à Paimboeuf ; 8 sont des pharmacies de quartiers, 3 des pharmacies de " passage » situées en galerie marchande.

Sur la zone miroir, 14 médecins généralistes ont accepté de participer dont 7 hommes et 7 femmes, répartis sur les communes de Nantes (7), Saint-Herblain (2), Sainte-Luce (1), Héric (2) et Nort-sur-Erdre (2). La moyenne d'âge est de 48,5 ans [32; 63]. 8 pharmaciens ont été enquêtés, dont 3 à Nantes, 1 à Saint-Herblain, 1 à Orvault, 1 à Sainte-Luce, 1 à Nort-sur-Erdre et 1 à Saffré ; 3 sont des « pharmacies de passage ».

\section{Présentation du terrain d'étude}

29 Les différences de recours à l'automédication dans la zone de l'aire d'emploi nazairienne (cf supra), nous a conduit à nous intéresser à ce territoire et à ce qui pouvait le singulariser des autres zones définies dans le cadre du projet AUTOMED, et notamment de l'agglomération nantaise.

\section{Le contexte urbain nazairien}

Avec 153596 habitants (données INSEE 2010), l'unité urbaine de Saint-Nazaire constitue la deuxième unité urbaine de Loire Atlantique derrière celle de Nantes (873 133 habitants, données INSEE 2010). Saint Nazaire fonde aujourd'hui son économie sur de grandes industries de la navale et de l'aéronautique.

31 De l'histoire de Saint-Nazaire, nous retiendrons la singularité de la construction de son maillage sanitaire et social. Dés la fin du XIX e siècle, sous l'impulsion des municipalités socialistes successives et du courant mutualiste, des réseaux de solidarités citoyens se mirent en place. Même si, depuis les années 1970, ces réseaux connaissent une très forte crise d'identité, leur existence même et l'interconnaissance entre les acteurs associatifs, sanitaires et sociaux permet encore aujourd'hui une bonne prise en charge des plus vulnérables (Lautrou 2008). Après l'arrivée de la gauche au pouvoir en 1981, l'histoire sanitaire de ce territoire a été également marquée par l'ouverture, malgré la forte opposition des médecins libéraux, de 1983 à 1986, de 3 Unités de Santé de Bases (USB) ${ }^{3}$ expérimentales (autrement dénommées CSI - centre de santé intégré), 2 à SaintNazaire, 1 à Trignac. Dans ces USB qui revendiquaient une "médecine globale ", tous les professionnels étaient salariés. De ce fait, le non recours au paiement à l'acte permettait de rémunérer l'activité hors soins (éducation, prévention...) et de pratiquer une médecine laissant plus de place à l'écoute et la discussion. L'accès aux soins était facilité par l'application du tiers payant, les actions de prévention étaient gratuites et les usagers étaient invités à participer au conseil d'administration de l'association 
(Coutant 1989). De retour au gouvernement, la droite a mis fin à l'expérience (sans qu'aucune évaluation ne soit réellement produite), mais celle-ci a eu une certaine influence sur le milieu médical et sur les patients : qu'ils aient ou non été usagers des centres de santé, les patients ont plus qu'ailleurs adhéré à l'option " médecin référent » proposé par leur médecin dès lors que celui-ci est connu comme étant un ancien acteur des centres de santé ${ }^{4}$.

\section{Une forte présence des populations fragiles}

L'aire d'emploi nazairienne est classé par l'INSEE comme un des «territoires les plus confrontés à la précarité " des Pays de la Loire contrairement à l'agglomération nantaise qui apparaît comme une " agglomération aux populations hétérogènes » dans cette même classification (Seguin, 2011). Le taux d'emploi salarié à durée limitée (CDD, Interim) sur l'aire d'emploi nazairienne est supérieur à $14 \%$ en 2011 contre $12,5 \%$ de moyenne nationale (INSEE, 2011). Sur notre zone d'étude, plusieurs quartiers bénéficient du Contrat Urbain de Cohésion Sociale (CUCS ${ }^{5}$ ), dont trois étudiés classés en Zone Urbaine Sensible (ZUS) avec des niveaux d'intervention prioritaire, la priorité 1 correspondant aux quartiers les plus nécessiteux d'interventions. Le quartier Ouest de Saint-Nazaire est classé en priorité 1 . Il a la part la plus importante de familles monoparentales et de population immigrée.

Figure 6 : Communes et quartiers étudiés

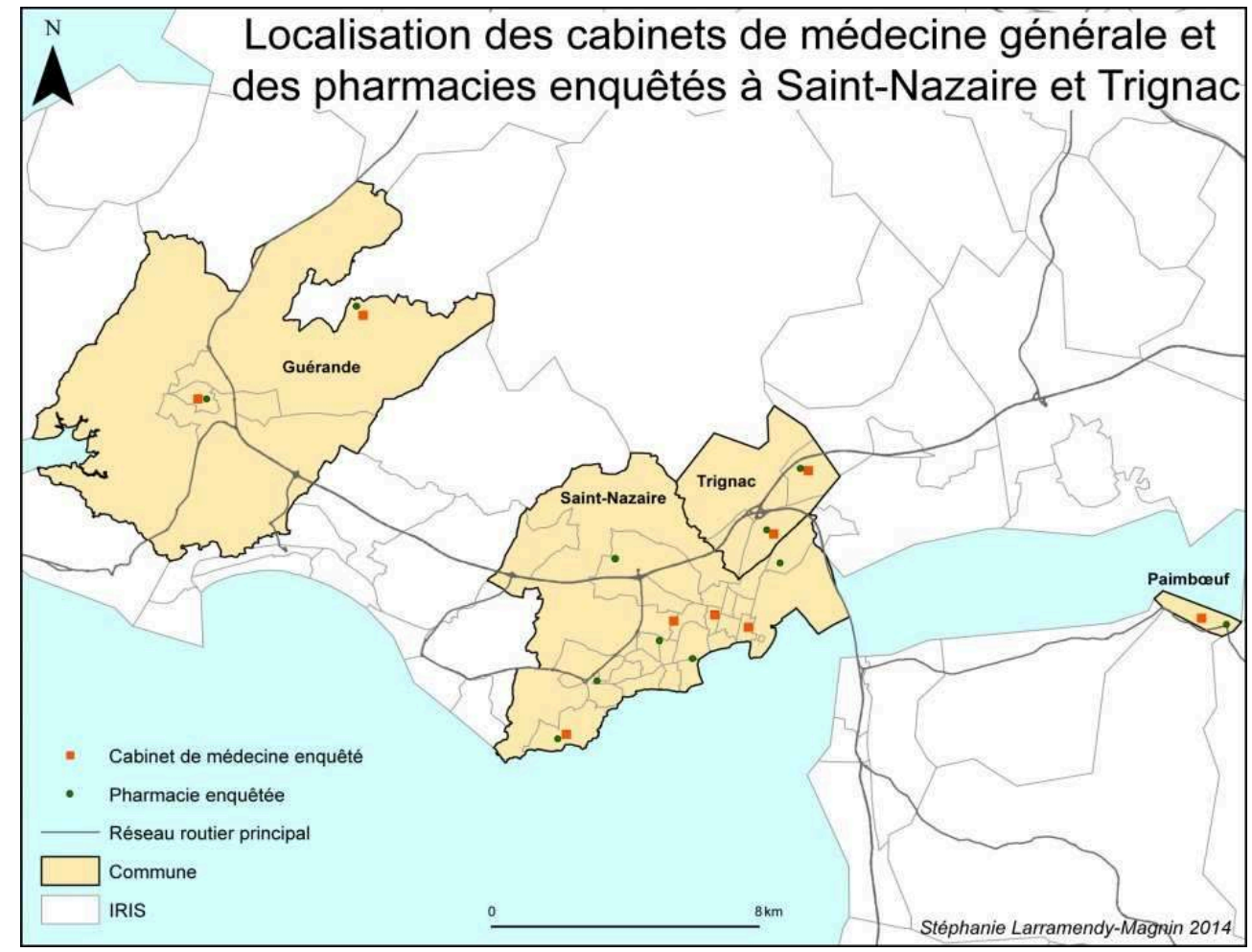

Ce sont les deux populations, avec les 16-24 ans, les plus en marge du marché de l'emploi à Saint-Nazaire, ce qui est corrélé avec le fort taux de chômage de ce quartier. Le quartier Certé à Trignac est classé priorité 2 et le quartier de Méan Penhoët à SaintNazaire en priorité 3. Les quartiers Certé et Méan/Penhoët présentent de forts taux d'emplois précaires. Ces quartiers sont situés à l'est de la ville dans la continuité des 
chantiers navals et sont peuplés, traditionnellement, des populations d'ouvriers et employés des grandes industries de l'Estuaire. Ils sont séparés l'un de l'autre par une voie ferrée. Le quartier Certé est séparé du bourg de Trignac par une voie express. Pris entre deux infrastructures de transport, le quartier est particulièrement enclavé. Le centre ville est le lieu d'une plus grande mixité sociale. Le quartier résidentiel de SaintMarc est lui le plus aisé de Saint-Nazaire.

34 Les Nazairiens disposent d'un plus faible niveau de formation post-baccalauréat que la moyenne départementale et d'un revenu médian inférieur à celui du département et de la France métropolitaine dans son ensemble. La part des Nazairiens concernés par le RSA $(5,9 \%)$ se situe au-dessus des taux départementaux $(3,0 \%)$ et nationaux $(3,8 \%)$. Le RSA activité seul est perçu par un peu plus de 700 bénéficiaires. Dans une ville marquée par l'importance de l'intérim dans les secteurs de l'industrie et du tourisme, ces bénéficiaires sont bien souvent des travailleurs précaires ou des travailleurs pauvres. Environ $8 \%$ de la population de la ville (allocataires et ayants droit affiliés au régime général) sont concernés par la CMU-complémentaire ${ }^{6}$ contre 3 à $4 \%$ au niveau départemental.

Du point de vue de la santé des populations, la situation locale est particulièrement défavorable en matière de mortalité prématurée (avant 65 ans) qui est supérieure de $29 \%$ à la moyenne régionale. La mortalité pour maladies directement liées à la consommation d'alcool est supérieure de $73 \%$ à la moyenne française. Cette consommation élevée d'alcool peut également expliquer pour partie les indicateurs défavorables en matière de pathologies mentales, d'affections cardiovasculaires, de suicide et de mortalité par accident de la vie courante (ORS Pays de la Loire et ADDRN 2013). Par contre, la situation épidémiologique actuelle est relativement proche de la moyenne nationale pour les cancers du sein et du côlon-rectum, et on observe globalement une bonne adhésion des Nazairiens aux programmes de dépistages organisés (ORS Pays de la Loire, 2013).

\section{Une certaine facilité d'accès aux soins primaires}

L'aire d'emploi nazairienne, à l'instar de l'agglomération nantaise, présente une forte densité de médecins généralistes (Conseil National de l'Ordre des médecins, 2013) par rapport à la moyenne régionale. Les distances à parcourir pour accéder à l'offre de soin dans l'agglomération nazairienne sont faibles, la population étant concentrée sur une langue de terre entre marais et littoral océanique.

37 A l'exception de 6 médecins installés à Saint-Nazaire et qui pratiquent une activité particulière justifiant des temps de consultation plus long (acupuncture, homéopathie, angiologie), tous les médecins généralistes de Saint-Nazaire, Guérande, Trignac et Paimboeuf sont en secteur 1 (pas de dépassement d'honoraire) ce qui limite le frein financier d'accès aux soins.

La Cité Sanitaire de Saint Nazaire a été implantée en lisière du Quartier Ouest dont les habitants bénéficient ainsi d'un accès rapide au service d'urgences et à la Permanence d'Accès aux Soins de Santé, service de soins pour ceux qui sont hors du droit commun (pas de couverture maladie). 


\section{Les facteurs contextuels influencent les pratiques}

39 L'analyse des entretiens nous a permis de mettre en avant des différences d'approche de l'automédication par les professionnels en fonction des données socio-économiques et culturelles, et de la construction des jeux d'acteurs dans les différents lieux d'exercice.

\section{Le poids des données socio-économiques et des différences interquartiers}

40 L'analyse des 49 entretiens sous le prisme du contexte socio-économique des quartiers ou communes d'implantation des cabinets médicaux et pharmacies met en évidence des différences de discours entre les professionnels selon qu'ils exercent au contact de populations favorisées ou défavorisées.

C'est le cas notamment des perceptions de politiques publiques telles que les déremboursements de médicaments qui peuvent encourager indirectement l'automédication. Depuis 2003, le gouvernement français a décidé du déremboursements d'un certains nombres de médicaments au "service rendu » jugé insuffisant pour justifier de leur prise en charge par la Sécurité sociale. Ces médicaments peu efficaces et qui n'ont pas été retirés du marché sont accessibles en pharmacie sans ordonnance. Le gouvernement espérait ainsi diminuer les dépenses de santé en réduisant le nombre de médicaments pris en charge et potentiellement le nombre de consultations, certains patients ne consultant plus leur médecin pour obtenir ces médicaments. Plus de la moitié des médecins (les 3/5èmes) estime qu'un certain nombre de médicaments sont inutiles, voire dangereux et que leur déremboursement leur donne un argument supplémentaire pour ne plus les prescrire. Le deuxième effet bénéfique des déremboursements, souligné par environ 1/4 d'entre eux, est qu'ils contribuent à préserver le système de soins français en améliorant les finances de la Sécurité sociale. Des nuances dans les discours apparaissent toutefois chez les médecins et les pharmaciens principalement implantés dans les quartiers en ZUS. Ils mettent en avant que les déremboursements des médicaments dits "de confort » défavorisent les moins fortunés et parlent d'une médecine à deux vitesses (notamment 8 médecins dont 6 situés en zus). En effet, si ces médicaments ne sont pas d'une grande efficacité, ils peuvent soulager des symptômes, apporter du confort à l'usager, ce qui, en matière de santé, devrait pouvoir être accessible à tous. L'exemple le plus fréquemment évoqué (5 médecins sur les 8 soulignant les inégalités de santé liées aux déremboursements) est celui du traitement des hémorroïdes où tous les médicaments de première ligne ont été déremboursés donc les médecins sont amenés à prescrire des anti-inflammatoires pour soulager leurs patients alors que ce traitement présente peut présenter de nombreux effets indésirables. Cet exemple fait d'ailleurs apparaître un deuxième effet négatif des déremboursements: des prescriptions contraintes par les difficultés économiques des patients. Les médecins ne pouvant, en effet, prescrire un traitement non remboursé puisqu'ils savent que leurs patients sont dans l'incapacité financière de l'acquérir, prescrivent plutôt des traitements remboursés mais plus forts avec plus de risque d'effets secondaires. Certains déremboursements peuvent donc finalement être contre-productifs à la fois pour le patient et pour la Sécurité sociale. Le témoignage suivant émane d'un médecin femme de 44 ans exerçant en ZUS à Nantes, à propos des médicaments déremboursés : "Je trouve que c'est un peu dommage parce que c'est toujours les mêmes qui n'y ont plus 
droit. Mais c'est vrai qu'il y en avait qui étaient vraiment inutiles. Il y en a d'autres qui sont quand même plus du confort et je ne vois pas pourquoi tout le monde n'y aurait pas droit. Quand on prend par exemple les médicaments contre les hémorroïdes, y en a plus aucun qui est remboursé. Enfin je trouve ça lamentable. Pourquoi les pauvres devraient payer... ça fait mal. C'est pas grave c'est vrai mais voilà quoi. Encore une médecine à deux vitesses. "

Les stratégies de vente des pharmaciens varient également en fonction du type de quartier où ils sont implantés. La différence se fait sur ce qu'ils nomment «le conseil » délivré à l'usager pour l'achat de médicaments hors prescription. Une gradation s'opère depuis les quartiers les plus défavorisés vers les plus favorisés en passant par les quartiers plus mixtes. Ainsi, à Trignac bourg, le pharmacien cherche rarement à vendre un médicament supplémentaire lorsque les patients se présentent avec une ordonnance (ce qui est fréquemment le cas par ailleurs). Dans les pharmacies situées dans des zones de passage ou des quartiers plus mixtes, les pharmaciens modulent leurs conseils en fonction du client et ne proposeront que « le produit le plus essentiel » à ceux qu'ils auront identifiés comme ayant de faibles revenus et mettront « leur plein conseil » avec la vente de deux ou trois produits " pour soigner au mieux » à ceux qu'ils jugeront en capacité financière d'acheter ces produits. Ces pharmaciens (3 sur 19) qui adaptent leur discours au contexte évoquent toujours la bonne connaissance qu'ils ont de leur clientèle, fondant ainsi leur raisonnement non pas sur des apparences mais sur la réalité perçue de leur environnement. Dans les quartiers ou communes favorisés (Guérande, Saint-Marc), les pharmaciens n'évoquent pas les freins financiers, qui de fait sont moins présents, et semblent pratiquer le même conseil pour tous. Ces stratégies sont mises en place avec l'intention louable de ne pas mettre la personne en difficulté, soit en la mettant face à son incapacité financière à acquérir ce qui lui a été présenté comme le traitement optimal pour ses symptômes voire, plus culpabilisant, pour ceux de ses enfants ; soit justement en l'incitant à dépenser plus qu'elle ne serait en capacité de le faire réellement Elles dessinent toutefois le début des inégalités de santé, avant même l'acte d'achat, au moment du conseil, le choix proposé n'étant pas le même en fonction du niveau supposé de revenu.

43 Les perceptions de l'automédication varient en fonction du contexte socio-économique du quartier et de la commune. Dans les communes et quartiers favorisés, les discours vont plutôt dans le sens d'une automédication choisie par des patients "capables " d'accéder à l'information et qui peuvent être responsabilisés. Dans les plus défavorisés, les professionnels mettent l'accent sur l'éducation des patients, l'information délivrée (Quartier Ouest ZUS en priorité 1 et Trignac Bourg). " Qu'ils se prennent en main c'est très bien, j'essaie de le privilégier, et de les éduquer " explique une médecin quadragénaire de Trignac bourg. Seuls les professionnels du quartier de Trignac Certé (ZUS en priorité 2) expriment, dans leur ensemble, une inquiétude vis-à-vis de l'automédication et sont plutôt défavorables à son recours par les patients. Le pharmacien évoque par exemple les « soucis » auxquels il a déjà été confronté, « c'est un potentiel danger » témoigne-t-il en parlant de l'automédication des usagers, et c'est ce qui l'inquiète. Ce jeune pharmacien d'une trentaine d'années rapporte déjà plusieurs cas relativement graves faisant suite à une automédication inadaptée.

44 Les niveaux socio-économiques et de formation de la population ne sauraient être les seules facteurs explicatifs d'une automédication perçue comme dangereuse par les professionnels. En effet, dans le Quartier Ouest, aux indicateurs socio-économiques 
pourtant plus défavorables l'automédication est perçue plus favorablement par les professionnels. Mais dans ce dernier quartier l'accès aux soins est favorisé à la fois par un accès plus élevé dans la population au bénéfice de la CMU-C (plus importante que dans le quartier Certé), par l'implantation d'une ancienne Unité de Soins de Base où exerce encore un médecin ayant participé au projet et où un lien fort s'est historiquement établi avec la population environnante (cf. précédemment) limitant certains freins d'accès aux soins (représentations, freins culturels...) et par la présence de la cité sanitaire à sa lisière avec un service des urgences et la PASS (Permanence d'accueil solidarité santé). Comme nous l'avons évoqué précédemment, le quartier Certé fait lui l'objet depuis quelques années d'aménagements urbains et bénéficie d'un réseau associatif dynamique. Alors pourquoi, ici plus qu'ailleurs, les médecins essaient ils de prévenir les risques de l'automédication avec une attention toute particulière pour les patients les plus fragiles? Une démographie médicale stagnante dans ce quartier en pleine rénovation où la construction de nouveaux logements sociaux attire de nouvelles familles, pourrait expliquer le recours à l'automédication de la population faute de disponibilité des médecins. Mais elle ne saurait expliquer la perception négative de cette pratique ici plus qu'ailleurs. Un élément explicatif avancé par les médecins rencontrés en entretien serait l'implantation historique d'ouvriers des industries du bassin nazairien dans ce quartier, des travailleurs qui occupent des emplois précaires souvent en intérim, l'offre fluctuant au grès des carnets de commandes des entreprises. Ces travailleurs pauvres ont néanmoins des revenus supérieurs au plafond fixé pour être bénéficiaire de la CMU. Un médecin témoigne que la plupart préfèrent acheter une boite d'antalgique à $1,90 €$ plutôt que de perdre une demi-journée de travail et $6,90 €$ (tiers payant) pour venir consulter, d'autant que la précarité de leur emploi les pousse bien souvent à refuser les arrêts de travail. Apparaît donc dans ce quartier ouvrier «traditionnel » une automédication subie, révélatrice d'une inégalité d'accès aux soins : trop de revenus pour bénéficier de la CMU, pas assez pour consulter. Cette automédication est dans un premier temps délétère du fait des retards de diagnostic. Dans un second temps, elle est source de iatrogénie, car pratiquée par des patients mal suivis et qui, lorsqu'ils vieillissent, développent des pathologies chroniques non diagnostiquées et découvertes lors d'accidents consécutifs à la prise de médicaments non adaptés à leur état de santé. Cette automédication est presque invisible puisque ces usagers ne consultent pas les médecins, n'achètent finalement que peu de médicaments (paracétamol, anti-inflammatoire). Ayant un emploi, ils n'entrent pas, ou peu dans le spectre des populations qui côtoient les travailleurs sociaux (qui concentrent leurs actions sur les populations les plus exclues, souvent sans emploi) et sont donc mal informés sur les réseaux d'entraide pourtant nombreux et structurés à Saint-Nazaire. Cette automédication subie révèle la fragilité d'une population active précaire qui n'a d'autre choix que d'être " autonome " pour la prise en charge de sa santé. C'est de cela dont témoigne un des médecins du quartier Certé : " actuellement, dans nos quartiers, nous en sommes où des personnes qui auraient absolument besoin d'un arrêt de travail refusent un arrêt de travail parce que pas envie de perdre une journée de travail. Donc à ce stade là on est aux limites de la précarité pour dire le mot. Donc le gars il a le choix entre acheter une boite de Doliprane par exemple et perdre une partie de sa demi-journée de travail pour avoir une consultation. Le choix il est comme ça, le plus souvent". 


\section{L'influence des croyances traditionnelles} patientèles des quartiers influencent diversement les pratiques et perceptions des professionnels de santé. Les croyances traditionnelles sont, elles répandues dans l'ensemble des couches sociales et apparaissent dans les pratiques des médecins qui en sont familiers. La question de ces croyances n'est pas anecdotique et s'inscrit dans l'opposition ancienne entre un discours savant qui tend à les nier ou à les condamner et un corpus d'observations relevant du folkore, comme le notaient déjà Loux et Morel en 1976 au sujet des savoirs et pratiques populaires des femmes dans la façon de prendre soin de leurs nourrissons. Mais pour ces auteures, la confrontation de ces regards permet d'expliquer certaines attitudes incomprises par les médecins.

Ici, les populations originaires de la région nazairienne sont particulièrement sensibles à la croyance traditionnelle des « vers ». Selon cette croyance (qui n'est pas spécifique à Saint Nazaire, mais se retrouve à proximité de nombreuses zones humides, ici la Brière), ces parasites toucheraient principalement les enfants, lors de la pleine lune, et seraient cause d'un grand nombre de symptômes, allant de l'insomnie aux maux de têtes en passant par la fièvre ou la toux. Les parents font alors souvent appel à des personnes supposées détenir des pouvoirs spécifiques, appelées conjureurs, rebouteux, magnétiseurs, parfois même avant toute consultation médicale. Les patients peuvent aussi en faire part à leur médecin et demander une prescription de Fluvermal, vermifuge accessible sans ordonnance mais remboursé à $65 \%$ sur ordonnance, et ce malgré l'absence de signes objectifs de contamination par des vers (troubles digestifs, prurit anal).

La question était posée aux médecins des demandes spécifiques de médicaments faites par les patients pour un motif différent de celui de la consultation. Ceci peut, en effet, s'apparenter à de «l'automédication sur ordonnance suggérée " puisque le diagnostic et le traitement sont donnés par le patient, même si cela requiert la complicité du médecin qui accepte de prescrire. Sur les 16 médecins enquêtés sur le territoire élargi de Saint-Nazaire, 14 ont évoqué spontanément les demandes de vermifuge. Une seule évocation est relevée sur les 14 médecins interrogés sur le territoire de Nantes élargi. Ces résultats suggèrent un poids plus important de ces croyances dans le bassin de Saint Nazaire que dans la région nantaise. L'approche et la réponse données sont alors différentes en fonction de l'origine géographique du médecin. Les médecins originaires de la région qui ont toujours vécu avec ces croyances sont plutôt tolérants même s'ils restent critiques, et vont accéder à la demande des patients. Un médecin de SaintNazaire originaire de la région témoigne : "C'est culturel donc... ce n'est pas à nous d'aller à l'encontre à partir du moment que ce n'est pas dangereux. Pourquoi pas ». A l'opposé, les médecins originaires de régions plus éloignées évoquent avec ironie ce phénomène " culturel », comme ce médecin exerçant à Saint-Nazaire mais originaire de l'Est de la France: «Quand ils ne sont pas conjurés ils bouffent à peu près tout ce qu'ils peuvent trouver parce qu'il faut se purger parce qu'ils ont des vers et que ça leur monte au cerveau. C'est typique de la région ». S'ils refusent la prescription lorsque rien n'est objectivable, ces derniers médecins concluent néanmoins que les patients arrivent toujours à s'en faire prescrire ou à s'en procurer, certains médecins en prescrivant très facilement et les pharmaciens étant totalement partie prenante dans le jeu. Apparaît donc ici le poids des croyances qui influencent à la fois les demandes des patients et les pratiques des médecins.

Revue francophone sur la santé et les territoires, Miscellanées 


\section{La construction des jeux d'acteurs}

48 thèmes sont abordés pour apprécier le positionnement des médecins par rapport à l'automédication. Le premier est celui de l'échange et de la discussion, le second celui du conseil délivré par le médecin (de l'information à l'éducation). Sur la thématique du conseil en général, les résultats diffèrent peu d'une zone à l'autre, avec un peu plus de $80 \%$ d'apparition de la thématique dans les deux zones. Dans le discours en tout cas, les médecins semblent donc majoritairement informer les patients sur la bonne utilisation des médicaments. Le terme " éduquer » apparaît toutefois plus souvent sur la zone de Saint-Nazaire (5/16) que sur la zone de Nantes (3/14). La thématique de l'échange et de la discussion apparaît dans 9 entretiens sur 16 réalisés sur la zone de Saint-Nazaire contre 6 sur 14 sur la zone de Nantes. Le terme « écouter » apparaît notamment dans 6 des entretiens sur la zone d'étude principale contre 3 sur la zone miroir. Il apparaît donc que les conseils sur les médicaments à utiliser en automédication sont plutôt apportés par les médecins sous forme de recommandations sur les bonnes pratiques sur la zone de Nantes alors qu'ils s'élaborent plutôt au cours d'échanges sur les pratiques des patients à Saint-Nazaire, dans un procédé à double sens comme l'explique cette médecin de Trignac Bourg : "si on n'entend pas le patient, et qu'on ne sait pas ce qui se passe à côté, il peut y avoir des bêtises de faites d'un côté ou de l'autre. Moi je trouve que c'est surtout un dialogue avec le patient et être capable de tout entendre sans se fâcher, le but c'est quand même de responsabiliser les gens »

grille d'entretien visait également à évaluer dans quelle mesure le médecin peut aider les patients à «s'automédiquer ». Encourager l'automédication revient pour les médecins à préconiser, voire prescrire, un traitement pour des récidives de symptômes qu'ils ont identifiés avec le patient. Tous les médecins préconisent la prise de Paracétamol mais certains vont beaucoup plus loin et mettent en avant la capacité de leurs patients à " gérer » leurs symptômes une fois l'information délivrée. Les pratiques ont été classées en deux catégories: une automédication encouragée "à minima ", c'est-à-dire simplement avec du Paracétamol et éventuellement un deuxième produit, et une automédication encouragée plus largement. Dans la zone de Saint-Nazaire, l'automédication est encouragée « à minima » dans 6 cas sur 16 et plus largement dans 10 cas sur 16 alors qu'à Nantes, elle est encouragée a minima dans 8 cas sur 14, plus largement dans 6 cas sur 14. L'automédication est donc encouragée de façon plus importante dans la zone de Saint-Nazaire, ce qui témoigne d'une plus grande volonté 
des médecins à autonomiser leurs patients et une plus grande confiance dans leur capacité à être autonomes.

Un certain fatalisme face au recours des usagers à l'automédication peut ressortir dans le discours de certains praticiens de la région nantaise (1 sur 2). Mais les positionnements sont très variés dans cette zone, avec des médecins qui intègrent aussi parfaitement ces pratiques à la leur (1/4). Sur la région de Saint Nazaire c'est la résonance collective des discours des médecins interrogés, quel que soit le type de quartier d'exercice, qui singularise ce territoire. Que les médecins soient pour ou contre l'automédication, il ressort globalement (14 sur 16) de leurs discours une certaine bienveillance et un souci de ne pas être dans le jugement, comme l'illustre le propos suivant d'un médecin de 52 ans de Saint-Nazaire : "Ce n'est pas constructif (ndr: de critiquer de façon négative l'automédication du patient), ce n'est pas thérapeutique et pour le coup c'est inadapté. Dans le temps de consultation ça n'a pas sa place. Enfin moi je trouve personnellement. On peut informer mais la critique etc. je ne vois pas... quand bien même c'est fait, c'est fait ».

Les médecins de l'agglomération nazairienne prennent donc le temps d'interroger l'automédication de leurs patients pour pouvoir les conseillers, les éduquer en fonction des pratiques de chacun d'eux. Ils leur permettent ainsi de s'autonomiser dans la prise en charge de leur santé selon un modèle participatif. A ces résultats fait écho l'histoire sanitaire et sociale singulière de l'agglomération nazairienne et notamment celle des centres intégrés de santé vue précédemment, suggérant l'héritage d'une "certaine philosophie» de la médecine sur ce territoire: pratiquer une médecine globale, associant soins et préventions. Cet état d'esprit s'est transmis aux nouveaux arrivants qui, avant de s'installer, ont côtoyé durant leur formation, les médecins de l'époque des centres intégrés de santé. Dans l'ancienne Unité de Soins de Base du quartier ouest, l'un d'eux témoigne : «je pense qu'on m'a donné le goût aussi peut être, au cours de mon stage d'interne de venir ici aussi ». Ainsi, à l'évocation des raisons qui les ont poussé à pratiquer à Saint-Nazaire, et dans ce quartier en particulier, le vocable des médecins militants de l'époque resurgit: «j'aime bien prendre mon temps pour faire la médecine, c'est vrai que l'exercice du cabinet ça correspondait tout à fait, parce que ici les médecins ils prennent le temps de bien écouter les patients de bien les voir, ce n'est pas la course à la consultation et puis le fait de pouvoir discuter avec les gens d'un point de vue de leur vie sociale, de leur vécu, c'est aussi intéressant ».

\section{Discussion}

\section{Liens entre recours à l'automédication et la relation médecin/patient}

Notre étude est basée sur l'analyse d'entretiens approfondis, dans une démarche qualitative sur un échantillon restreint ( 49 personnes). Et même si le choix des terrains d'étude repose sur une analyse spatiale faisant appel à des méthodes quantitatives, il serait dangereux de vouloir faire une approche écologique entre les résultats de l'étude SSPSM et ceux issus des entretiens avec les professionnels de santé ; c'est-à-dire entre le faible taux de recours à l'automédication de la population et les pratiques et perceptions des médecins sur le territoire d'étude. Néanmoins, le fait que des différences de perception et de rapport à l'automédication apparaissent sur des territoires eux-mêmes différenciés par des taux de recours et d'évocation de l'automédication significativement distincts (cf: choix du terrain d'étude) conduit à 
s'interroger sur la façon dont une relation médecin/patient basée sur la confiance et l'écoute et sur la façon dont l'inscription dans un contexte local de cette relation peuvent influencer les pratiques d'automédication de l'individu. Les entretiens avec les pharmaciens apportent beaucoup d'informations sur les relations médecins/patients, les usagers confiant leurs doléances à ce sujet au comptoir de l'officine. Si aucun retour négatif n'a été formulé dans la zone de Saint-Nazaire, soulignant plutôt le lien étroit entre médecins et patients, il en va tout autrement dans l'agglomération nantaise. Le témoignage de deux pharmaciens exerçant dans la même officine en périphérie nantaise apporte un éclairage sur le lien possible entre la relation médecin/patient et l'automédication :

" $B$ : les gens se tournent de plus en plus vers autre chose.

- A : vers des médecines plus douces, parallèles.(...) Les gens nous parlent qu'ils vont voir des ostéopathes, des homéopathes, des naturopathes, des étiopathes, des faciothérapeutes. On a un naturopathe qui s'est installé tout près de chez nous et il y a beaucoup de gens qui s'arrêtent pour nous demander où il est installé.

- B: des gens qui se tournent vers autre chose, peut être parce qu'ils n'ont pas de répondant je ne sais pas (...) ils ne se sentent pas écoutés. Donc ils essaient autre chose même jusqu'à commander sur internet des trucs.

- Et comment vous expliquez cela?

- A : Par une écoute qu'ils ne trouvent plus chez le jeune médecin. C'est la rentabilité, la vitesse.

- B : Ils nous disent, « on n'est pas écouté ». (...) Ensuite y a peut-être une histoire de spécialisation de plus en plus pointue des médecins à l'hôpital entre autre, qui vont dans une direction et qui ne se préoccupent pas du reste. Ils sont dans leur direction qu'ils maitrisent et si le patient parle d'autre chose, le reste on ne l'écoute pas. ».

Cette relation "déçue " du patient avec son médecin, également évoquée par Sylvie Fainzang dans ses travaux (2012), peut sans doute expliquer en partie, dans une perspective inversée, la spécificité du bassin nazairien par une relation privilégiée entre soignant et soigné. Les bons taux de recours aux dépistages organisés des cancers du colon et du sein objectivés dans le diagnostic santé de Saint-Nazaire sont des indicateurs de l'adhésion et donc sans doute de la confiance de la population dans les préconisations du médecin et dans le système de soins local. Les populations défavorisées ont en effet habituellement moins recours à ces dépistages pourtant gratuits que les populations socio économiquement favorisées (Bertolotto et al. 2003).

\section{Surreprésentation des femmes médecins dans l'échantillon}

L'étude qualitative fait ressortir que les femmes médecins prennent plus en considération les pratiques d'automédication de leurs patients que les hommes. La littérature montre d'une part que les femmes sont généralement plus attentives à leur santé que les hommes et consultent plus fréquemment leur médecin (Aliaga, 2002) et d'autre part que le recours à l'automédication semble concerner préférentiellement les femmes, notamment celles d'âge actif (Raynaud, 2008 ; Laure, 1998 ; Johnson \& Pope, 1983). En prenant garde à ne pas donner dans l'interprétation essentialiste, il est toutefois possible de se demander si l'expérience personnelle de la femme médecin visà-vis du soin et en particulier de l'automédication pourrait influencer ses pratiques? L'hypothèse serait ici que la femme médecin appréhende plus spontanément 
l'automédication puisqu'elle y a plus souvent recours (pour elle mais aussi dans le cadre de la sphère domestique et familiale où son rôle reste prépondérant, cf. Lapeyre et Lefeuvre, 2005) et donc a pu l'intégrer comme une donnée courante des pratiques de soins et que, de ce fait, elle l'interroge plus facilement. Cette interrogation nécessiterait une approche ethno-sociologique ce qui n'est pas la nature de ce travail mais se pose néanmoins la question de la surreprésentation des femmes dans notre échantillon. En Loire Atlantique seulement 46\% des médecins généralistes sont des femmes en 2012 (Romestaing et Le Breton-Lerouvillois 2012) contre 68,5\% dans notre échantillon de la zone de Saint-Nazaire et $50 \%$ dans notre échantillon nantais. Mais même si ce panel de médecins n'a pas vocation à être représentatif de la population médicale, le déséquilibre entre les deux échantillons peut induire un biais d'interprétation dans l'analyse du croisement des données. Les taux de refus étaient proches sur les deux zones, $33 \%$ sur la zone de l'aire d'emploi de Saint-Nazaire et $32 \%$ sur la zone de Nantes et agglomération. Mais ces taux de refus sont très différents selon le genre de la personne sollicitée. Dans la zone focus (St Nazaire), sur les 12 médecins hommes contactés qui ont donné une réponse, $58,3 \%$ ont refusé de participer à l'étude contre $26,6 \%$ des 15 femmes appelées. Dans la zone miroir, sur les 11 médecins hommes contactés et qui ont donné une réponse, $46,1 \%$ ont refusé l'étude contre $25 \%$ des 9 femmes contactées sur Nantes. Ces taux de refus, même s'ils déséquilibrent notre échantillon, confortent l'hypothèse d'un plus grand intérêt porté par les femmes à la problématique de l'automédication que les hommes.

57 Le biais induit par le déséquilibre des sexes ratio est également lié à la réalité du contexte étudié: la commune de Trignac, où tous les médecins ont été enquêtés, compte $80 \%$ de femmes médecins. De plus, sur huit médecins rencontrés exerçant dans des quartiers en politique de la ville $75 \%$ étaient des femmes. Nous ne disposons pas ici de données générales sur la démographie médicale dans les quartiers défavorisés mais ces constats posent la question d'une sensibilité plus grande des femmes médecins pour une médecine où les problématiques sociales des patients revêtent une grande importance dans leur prise en charge médicale. Le Baromètre santé INPES 2011 fait état d'un plus grand investissement des femmes médecins dans les questions d'éducation (INPES 2011). Ces dimensions d'éducation, de prévention, se retrouvent dans la pratique d'une "médecine globale» qui fait écho à la notion de communauté de pratiques en lien avec l'expérience des UBS nazairiennes. Cette notion, particulièrement développée au Canada, centre l'exercice médical non pas sur la maladie mais sur le patient (McWhinney 1980). Elle a été abordée dans nos entretiens par des médecins travaillant dans les contextes socio-économiques les moins favorables, l'importance de la dimension psychosociale apparaissant sans doute plus facilement au sein des populations les plus en difficultés. Les femmes médecins, plus que les hommes, pourraient être attirées par un exercice dans ces quartiers perçus non pas comme plus favorables mais comme plus nécessiteux d'actions d'éducation et de prévention. Ce constat a pu être dressé par ailleurs dans des études plus générales (Sicart, 2000), mais ici la nouveauté est le lien qui semble apparaître entre une manière d'aborder l'exercice de la médecine (influencée par le genre), un contexte, et un rapport à une pratique : l'automédication. Il est probable qu'une distinction effectuée, cette fois ci non plus sur le sexe mais sur l'âge, produirait des constats similaires. 


\section{Conclusion}

L'étude des pratiques et perceptions de l'automédication dans un contexte local avec, en miroir l'éclairage d'un contexte urbain différent a permis de mettre en lumière à la fois la diversité des pratiques en réponse à la singularité de chaque territoire et le nécessaire éclairage des discours des professionnels, par les données socio-territoriales. Ainsi, nous avons pu mettre en avant le poids du contexte socio-économique, des croyances traditionnelles et de l'histoire sanitaire du territoire dans les pratiques et perceptions des médecins vis-à-vis de l'automédication.

Sur la zone de Saint-Nazaire, les dynamiques sanitaires et sociales sont ancrées depuis longtemps dans une volonté politique et citoyenne de solidarité, de travail en réseau et de proximité avec une population fragilisée par la rudesse et la labilité des emplois qu'elle occupe. Il en découle un lien privilégié entre le médecin et le patient dans lequel s'inscrit notre problématique, où la prise en compte de l'automédication par les praticiens s'insère dans une démarche globale centrée sur le patient et non la pathologie. La singularité de cette relation pourrait être l'une des explications du faible recours à l'automédication des usagers dans cette zone. Néanmoins, la limitation de cette pratique ne saurait être, dans le contexte nazairien, expliquée par le seul lien de confiance marqué entre soignants et soignés. Les faibles niveaux socio-économiques d'une partie de la population de ce bassin d'emploi pèsent sans doute aussi sur le choix du recours à l'automédication.

Ainsi l'automédication dont le recours dépend de l'expérience, l'histoire et le contexte de l'individu (Fainzang 2012) ne pourra être analysée sans tenir compte des données spatiales entourant soignant et soigné, là où se jouent les liens sociaux et où s'ancre le vécu et l'histoire des individus.

61 C'est également la mise en perspective des discours et du contexte local qui nous a permis de mettre en avant une automédication subie, loin d'une volonté revendiquée d'autonomie des usagers. Dans un quartier accueillant une grande part de travailleurs pauvres, cette pratique s'impose en effet face à leurs difficultés économiques d'accès aux soins. L'inquiétude des professionnels de santé témoigne des dangers d'une automédication pratiquée à défaut de consultation médicale.

Ce travail ouvre donc de nouvelles perspectives d'approche sur l'étude géographique du recours à l'automédication, une approche qui replace les individus dans leur contexte, au cœur des dynamiques sociétales qui les entourent et auxquelles ils participent.

\section{BIBLIOGRAPHIE}

Aliaga, C. 2002, Les femmes plus attentives à leur santé que les hommes. Insee première , ${ }^{\circ} 869$ 
Bertolotto, F., Joubert M., Leroux M., Ruspoli S., Ancelle-Park R., Jestin C., Albi N, et SanchoGarnier H., 2003, « Facteurs sociaux de l'absence de participation aux campagnes de dépistage organisé du cancer du sein ». BEH 4: 24-26.

Charlier B., Henri F., 2004, Démarche d'évaluation, communauté de pratique et formation professionnelle, Schwezerische Zeitsschrift für Bildungswissenschaften 26 2, S. 285-304.

Conseil National de l'Ordre des médecins, 2013, La démographie médicale en région Pays de La Loire . http://www.conseil-national.medecin.fr (consulté le 12/07/2014)

Coulomb A, et Baumelou A., 2007. Situation de l'automédication en France et perspectives d'évolution, France: Ministère de la santé et de la protection sociale. http:// www.ladocumentationfrancaise.fr/var/storage/rapports-publics/074000030/0000.pdf (Consulté le $25 / 09 / 2014$ )

Coutant D., 1989, Hier, une médecine pour demain: le Centre de santé de Saint-Nazaire . Paris: Syros/ Alternatives.

Coutinet N., et Abecassis P., 2007, Le développement des médicaments d'automédication : enjeux pour les firmes, les institutions de régulation et les consommateurs . http://halshs.archives-ouvertes.fr/ halshs-00198986 (Consulté le 25/09/2014)

Deloitte / Harris Interactive, 2013, Les Français et le système de santé : prise de conscience d'un changement inévitable - Baromètre Santé 2013. http://www.harrisinteractive.fr/news/2013/ CP_HIFR_Deloitte_04042013.pdf (Consulté le 25/09/2014)

Fainzang, S., 2006, « Transmission et circulation des savoirs sur les médicaments dans la relation médecin-malade ». in J. Collin, M. Otero \& L. Monnais, Le médicament au cœur de la socialité contemporaine. Regards croisés sur un objet complexe , Les Presses de l'Université du Québec, 267-79.

Fainzang S., 2012, L'automédication ou Les mirages de l'autonomie . Paris: Presses universitaires de France.

Fleuret S., Séchet R., 2006, «Spatialité des enjeux de pouvoir et des inégalités : pour une géographie sociale de la santé » in Séchet R., Veschambre V., Penser et faire la géographie sociale: contributions à une épistémologie de la géographie sociale . Rennes: Presses universitaires de Rennes.

Gautier A., 2011, Baromètre santé médecins généralistes 2009 . Institut national de prévention et d'éducation pour la santé (France), Saint-Denis: INPES éd.

INSEE, Observation sociale des territoires de la Loire-Atlantique , Cahier départemental, décembre 2011, http://www.insee.fr/fr/insee_regions/pays-de-la-loire/themes/infostat/is430/ cahier_départemental_44.pdf (Consulté le 23/07/2014)

IPSOS/CNAM, 2015, Le rapport des Français et des Européens à l'ordonnance et aux médicaments http:// www.ameli.fr/fileadmin/user_upload/documents/ Le_rapport_des_Francais_et_des_Europeens.pdf (Consulté le 25/09/2014)

Kearns R., Moon G., 2002, From medical to health geography: novelty, place and theory after a decade of change Progress in human geography , 26/5 : 605-625

Lapeyre, N., \& Le Feuvre, N., 2005, Féminisation du corps médical et dynamiques professionnelles dans le champ de la santé. Revue française des affaires sociales , (1), 59-81.

Laure P., 1998, « Investigation on self-medication: from disease to performance ». Thérapie 53 (2): 127-35. 
Lautrou, P-Y. 2008, « Entretien avec Daniel Sicard, Les Nazairiens n'ont pas de nostalgie » L'EXPRESS , janvier http://www.lexpress.fr/region/daniel-sicard-les-nazairiens-n-ont-pas-denostalgie_473645.html (Consulté le 25/09/2014)

Loux, F., \& Morel, M. F., 1976, L'enfance et les savoirs sur le corps: Pratiques médicales et pratiques populaires dans la France traditionnelle. Ethnologie française, 309-324.

McWhinney, Ian R. 1980, « La définitions de la médecine globale ». Canadian Family Physician 26: 1458.

ORS Pays de la Loire, et ADDRN, 2013, Observatoire de la santé des Nazairiens .

Pestiaux D., (Coordination rédactionnelle de la traduction en français), 2002, La définition européenne de la médecine générale-médecine de famille . Europe, Wonca, Centre Universitaire de Médecine Générale, UCL, Bruxelles, Belgique. http://www.snemg.fr/IMG/pdf/ Definition_Europeenne_de_la_Medecine_Generale_Wonca_Europe_2002.pdf (Consulté le 25/09/2014)

Picheral H., 2001, Dictionnaire raisonné de géographie de la santé . Montpellier, France: Université Montpellier III/GEOS.

Pouillard J., 2001, «L'automédication ». Rapport adopté lors de la session du Conseil national de l'Ordre des Médecins de Février . http://www.conseil-national.medecin.fr/sites/default/files/ automedication.pdf (Consulté le 25/09/2014)

Raynaud, Denis. 2008. «Les déterminants du recours à l'automédication ». Revue française des affaires sociales 1 (1): 81-94.

Romestaing P., Le Breton-Lerouvillois G. 2012. Atlas de la démographie médicale en France, Situation au $1{ }^{\text {er }}$ janvier 2012 - Tome II . Ordre National des Médecins. http://www.conseilnational.medecin.fr/sites/default/files/atlas2012_tome2.pdf (Consulté le 25/09/2014)

Schweyer, F. X., 2004, Les territoires de santé et la médecine libérale. Les enjeux d'une convergence. Lien social et Politiques-RIAC , 52, 35-46.

Seguin S., Observation sociale des territoires en Loire-Atlantique : précarité marquée au nord et dans la région nazairienne , 2011, INSEE, http://www.insee.fr/fr/themes/document.asp?ref_id=18119 (consulté le 12/04/2014)

Sicart D., 2000, Les médecins au $1^{\text {er }}$ janvier 2000 , Paris : Ministère de l'Emploi et de la Solidarité. Thay S., 2013., Parle-t-on d'automédication lors des consultations de médecine générale ? Enquête par observation directe auprès de 126 médecins de Loire-Atlantique et Vendée en 2012. Nantes: Faculté de Médecine.

Ventelou B., Rolland S., 2009, «Évaluation de l'implication des médecins généralistes libéraux dans le bon usage des médicaments ». Santé Publique 21 (2): 129-38.

Wennberg J.E., Gittelsohn A., 1973, Small area variations in health care delivery, Science, vol 14, $\mathrm{n}$ o 182 1102-1108

\section{NOTES}

1. Une aire urbaine ou « grande aire urbaine » est un ensemble de communes, d'un seul tenant et sans enclave, constitué par un pôle urbain (unité urbaine) de plus de 10000 emplois, et par des communes rurales ou unités urbaines dont au moins $40 \%$ de la 
population résidente ayant un emploi travaille dans le pôle ou dans des communes attirées par celui-ci. Le zonage en aires urbaines 2010 distingue également les "moyennes aires" où le pôle urbain est de 5000 à 10000 emplois, et les "petites aires » où le pôle est de 1500 à 5000 emplois.

2. Sur cette zone les femmes sont surreprésentées par rapport à la moyenne départementale. Ce point sera discuté dans la troisième partie.

3. Les Unités Sanitaires de Bases résultaient de la transformation de 3 cabinets médicaux de médecins dans le cadre du Réseau Sanitaire expérimental généralistes. La budgétisation fut rendue possible par la loi du 19 janvier 1983 et ses décrets d'application de juin 1984.

4. Selon les acteurs locaux rencontrés dans le cadre de nos entretiens.

5. Le contrat urbain de cohésion sociale est un contrat passé entre l'Etat et les collectivités territoriales qui engage chacun des partenaires à mettre en œuvre des actions concertées pour améliorer la vie quotidienne des habitants dans les quartiers connaissant des difficultés (chômage, violence, logement...).

6. La CMU-C donne droit à une prise en charge des dépenses de santé à hauteur de $100 \%$ des tarifs de la sécurité sociale. Un revenu mensuel

7. Le terme communauté renvoie ici à l'idée de l'existence d'une communauté professionnelle (Charlier et Henri, 2004). Wennberg et Gittelsohn, avancent d'une part que le style de pratique médicale est le produit de l'éducation et de l'apprentissage des médecins et d'autre part que le contexte local, qui s'ajoute à l'incertitude et à la singularité de la décision médicale, pousse les médecins à une simplification dans leurs prises de décision, les conduisant à opter pour l'alternative la plus sûre et la plus confortable. Cela revient, en fait, à se conformer au standard local et à ce qui est accepté comme tel par tous

\section{RÉSUMÉS}

Les pouvoirs publics français, dans un souci de maîtrise des dépenses de santé, encouragent le recours à l'automédication de la population. Les comportements d'auto-soin, qui consistent à consommer de sa propre initiative un médicament sans consulter un médecin, placent ce dernier au cœur d'un antagonisme entre la volonté d'autonomie des usagers confrontée à celle d'encadrement des pouvoirs publics et l'obligent à se positionner face à des pratiques dont par essence il est exclu par le patient. Cette étude cherche à comprendre, par la méthode de contextualisation des discours, l'influence du milieu géographique dans les positionnements et comportements des praticiens vis à vis de l'automédication. Elle a été réalisée à partir d'entretiens semi-directifs, selon des grilles élaborées conjointement avec des sociologues, des médecins et des géographes, auprès de 30 médecins généralistes et 19 pharmaciens des agglomérations nazairienne et nantaise. Les résultats de ce travail suggèrent l'importance de la prise en compte des données territoriales entourant soignant et soigné dans l'analyse des comportements des professionnels de santé et dans de nouvelles perspectives d'approche du recours à l'automédication. 
The French public authorities encourage the population to resort to self-medication to control health expenditure. Since risk may occur from this practice, general practitioners and pharmacists have been appointed to be the "guarantors" of a safe use for self-medication. Selfcare behaviours, implying the consumption of medication without consultation, put the practitioner in an antagonistic situation between the patients' will to be self-reliant in care use on the one hand, and the frame imposed by the public authorities on the other. This demands of the professionals that they adopt for themselves a position on such self-care practices in which they are excluded by the patient. This study aims at understanding the influence of the geographical environment on the professionals' position and behaviour in relation to selfmedication examined through the method of contextual speech. Semi-structured interviews have been conducted with 30 practitioners and 19 pharmacists from the Nantes and Saint-Nazaire areas based on questions defined by sociologists, doctors and geographers. The results of this study suggest that spatial data are salient to analyzing health professionals' behaviour in relation to this concern of patient's self-medication.

The interviews aimed at determining the professionals' perceptions with respect to the selfmedication of their patients in terms of whether they have encouraged or on the contrary, attempted to prevent these behaviours through arguments in favour of the public policies promoting self-medication. The questions deal with the professionals' career, the socio-economic characteristics of the patients and the motivations for their choice of place of work so as to link practices and behaviours with places. The simultaneous study of two different urban areas enables the analysis to distinguish the effects of context from geographical invariants. The main case study was Saint Nazaire with the case of Nantes used as a mirror to enable the identification of similarities or differences. Both case studies rely on a spatial analysis of data issued of the same program: the ANR Auto-med. The choice of Saint Nazaire as the main case study is based on two observations. First, the patients' rate of self-medication declared in a survey conducted within the ANR Auto-med program appears to be lower in Saint Nazaire than that of the regional area. Secondly, an investigation of a panel of medical consultations on the frequency of selfmedication mentioned by the doctor or the patient again indicates that the area of Saint Nazaire is below the average (tab.1).

Local socio-economic conditions, culture and history influence the practices of the health professionals. The socio-economic status of the patients influences the attitude to them of the doctors, for example, in terms of their medical costs. The professionals located in the most deprived districts underline the inequalities in health generated by the de-listing of drugs known as "of comfort" from those whose costs will be reimbursed to the patient. In this context, the pharmacists seem to offer advice according to their customers' supposed purchasing power. The health professionals located in a district concentrating a large number of poor workers express a certain concern with respect to the inequality of access to care. In this case, self-medication is an essential part of health-care behaviour because even if people provide a prescription, they are not necessarily reimbursed for certain kinds of drugs.

The medical and social history of the Saint-Nazaire area has shaped the relations between patients and doctors such that a distinct community of professional practice has appeared in this area. This community is defined by a global approach towards the patient's health, a broader concern with self-medication, and a significant emphasis given to listening and discussion during the consultations. Thus, the advice on drugs is simply part of the global relations between the patient and the GP or the pharmacist whereas in the mirror area of Nantes, this advice is delivered more as a recommendation of good practice from the "expert" to the non-expert. Interviews with pharmacists in Nantes suggest that self-medication offers a therapeutic alternative in response to a lack of listening that is prevalent among the doctors of the area. The doctors are influenced in their practices by perceived local beliefs about health. If the doctors are also native to their place of work and have always been embedded in such sets of beliefs, they are 
more tolerant than their fellow-doctors towards these beliefs and indeed will sometimes accept being guided by their patients when writing the prescription (although sometimes they may just add a placebo to seem to concur with the patient). The patients' resort to self-medication is neither perceived nor accompanied in a homogeneous way from one city to another or from one district to another. In the area of Saint Nazaire, medical and social interventions have been anchored for a long time in a political and social tradition of solidarity, networking and proximity with a population weakened by the roughness and the liability of working conditions. Consequently, this situation strengthens even further the relationship between doctor and patient having thus a strong influence on self-medication. These results suggest the importance of taking into account the territorial context in the analysis of the health professionals' behaviours opening new opportunities for studying self-medication.

INDEX

Mots-clés : automédication, comportements, territoires

\section{AUTEURS}

STÉPHANIE LARRAMENDY

Medecin Généraliste, DMG, Université de Nantes

SÉBASTIEN FLEURET

Directeur de recherche CNRS en géographie, UMR ESO 6590 